\title{
EFEITO DO MATRICONDICIONAMENTO INTEGRADO COM CONTROLE QUÍMICO E BIOLÓGICO NA EMERGÊNCIA DE PLÂNTULAS E NA PRODUTIVIDADE DE MILHO
}

\author{
CLAUDINEI ANDREOLI ${ }^{1}$, RAMIRO VILELA DE ANDRADE ${ }^{2}$
}

\author{
'Pesquisador Embrapa Soja. Caixa Postal 231, CEP. 86001-970 Londrina, PR. E-mail: andreoli@cnpso.embrapa.br \\ (autor para correspondência). \\ ${ }^{2}$ Pesquisador, Embrapa Milho e Sorgo. Caixa Postal 157, CEP. 35701-970 Sete Lagoas, MG.
}

Revista Brasileira de Milho e Sorgo, v.2, n.3, p.132-142, 2003

\begin{abstract}
RESUMO - Uma das principais causas da baixa produtividade de milho (Zea mays L.) é a qualidade de semente, que afeta o estande inicial e a população de plantas. O objetivo deste trabalho foi avaliar os efeitos da qualidade da semente e da integração do condicionamento matricial (MC) com o tratamento químico $\left(\right.$ Captan $\left.^{\circledR}\right)$ e biológico Kodiak ${ }^{\circledR}$ (Bacillus subtilis) na emergência de plântulas e na produtividade de milho, nas condições tropicais de Sete Lagoas, MG, e de estresses de frio, em Ponta Grossa, PR. Dois experimentos foram conduzidos na Embrapa Milho e Sorgo, em Sete Lagoas, em 1996 e 1998, e um em Ponta Grossa, PR, em 1997. Sementes de milho híbrido foram condicionadas com Micro-Cel E na presença de B. subtilis (600 mg kg-1 de sementes), B. subtilis + Captan ${ }^{\circledR}$ e ácido giberélico $\left(\mathrm{GA}_{4+7} 100\right.$ $\mu \mathrm{M}$ ). Dois lotes de sementes, com germinação de $95 \%$ e $85 \%$, foram condicionados à razão de 20: 5: 20 , a $22^{\circ} \mathrm{C}$, por 48 horas, com MC e na presença de Bacillus. Os parâmetros estudados foram: emergência de plântulas aos 10 e 20 dias, estande final, índice de velocidade de emergência (IVE), número de espigas ha ${ }^{-1}$, produção de grãos e de espigas ha ${ }^{-1}$. A qualidade inicial do lote de sementes foi fundamental no estabelecimento do estande e na produtividade de milho; a integração do MC com o tratamento químico e biológico beneficiam a qualidade da semente e a produtividade de milho, especialmente em condições de estresse. O condicionamento com $\mathrm{GA}_{4+7}$ não favoreceu a produtividade de milho e a integração do condicionamento matricial (MC) com o tratamento biológico (Bacillus) mostrou-se efetiva em aumentar o vigor das sementes de baixa qualidade.
\end{abstract}

Palavras-chave: Bacillus, vigor, matricondicionamento, germinação, priming e biopriming.

\section{MATRICONDITIONING INTEGRATED WITH CHEMICALAND BIOLOGICAL SEED TREATMENTS TO IMPROVE CORN STAND ESTABLISHMENTAND YIELD}

\begin{abstract}
One of the main reasons of low yield of corn (Zea mays L.) is seed quality which affects initial stand and plant density. The main objective of this work was to study seed quality and the integration of matriconditioning with chemical and biological seed treatments (Bacillus) on seedling emergence and yield of corn, under tropical conditions of Sete Lagoas, MG and under stressful conditions of Ponta Grossa, PR. Two trials were carried out at Embrapa Milho and Sorgo, Sete Lagoas, MG, in 1996 and 1998 and a third one was conducted at Embrapa SNT in Ponta Grossa, PR, in 1997. Seeds of hybrid corns were conditioned in the presence of Bacillus $\left(600 \mathrm{mg} \mathrm{kg}^{-1}\right.$ of seed) Bacillus $+\mathrm{Captan}^{\circledR}$ and $\mathrm{GA}_{4+7}(100 \mu \mathrm{M})$. Two seed lots with germination of $95 \%$ and $85 \%$ were conditioned with $\mathrm{MC}$ and with $\mathrm{MC}+$ Bacillus
\end{abstract}


at a rate of 20:5:20, at $22^{\circ} \mathrm{C}$ for 48 hours. Data were recorded for seedling emergence at 10 and 20 days, final stand, emergence rate, ear number ha ${ }^{-1}$, ear and grain yield per hectare. Initial seed quality affected the establishment and yield of corn; the integration of MC with chemical and biological seed treatments was effective in ameliorating seed quality and yield of corn, especially under stress conditions. Grain yield of corn did not respond to seed matriconditioning treatment with $\mathrm{GA}_{4+7}$ in Sete Lagoas, $\mathrm{MG}$ and the integration of matriconditioning with biological seed treatment was effective in improving the quality of seed lots.

Key words: Bacillus, vigor, matriconditioning, germination, priming and biopriming.

O Brasil, ao lado dos Estados Unidos da China, é um dos maiores produtores mundiais de milho; entretanto, ainda apresenta baixa produtividade. Uma das principais causas dessa baixa produtividade é a qualidade da semente, a qual afeta o estande inicial e a população de plantas (Andreoli et al., 2002).

Nas últimas décadas, o tratamento de précondicionamento tem sido prática comum em sementes de hortaliças (Khan, 1992; Andreoli \& Khan, 2000), especialmente para aumentar a tolerância ao frio (Khan et al., 1995; Andreoli \& Khan, 1999). Em adição ao condicionamento osmótico, forças matriciais sem propriedade osmótica (como vermiculita, calcinita e Micro Cel E) podem ser utilizadas para controlar a hidratação das sementes (Kubik et al., 1988; Khan, 1992). Kubik et al. (1988) e Taylor et al. (1988) introduziram o termo "solid matrix priming" (SPM) para o pré-condicionamento, visando acelerar e uniformizar a germinação. "Biopriming", uma combinação de condicionamento com controle biológico associado à semente, tem sido efetivo no controle de Pythium ultimum em milho doce (Callan et al., 1990; 1991). Andreoli \& Khan $(1993 ; 1999)$ mostraram a eficiência do condicionamento combinado com GA em sementes de mamão, pimentão e tomate. Recentemente, Andreoli (2001) e Andreoli \& Khan (2000) combinaram o condicionamento matricial com o tratamento químico e biológico, para beneficiar o estande e a produtividade de várias culturas. Outra tecnologia para controlar a hidratação da semente foi designada "drum priming" (Rowse, 1996; Warren \& Bennett, 1997). Taylor et al. (1998) elaboraram interessante revisão sobre diferentes métodos de condicionamento de sementes. Apesar de inúmeras informações disponíveis sobre condicionamento, ainda existem obstáculos para a aplicação em escala comercial para sementes de grandes culturas e pouca informações sobre o condicionamento em sementes de milho, especialmente nas condições do Brasil.

Vários produtos têm sido utilizados em combinação com o condicionamento matricial, entre eles, o ácido giberélico (GA), que envolve a degradação da parede celular do endosperma das sementes de alface, beringela, pimentão e tomate (Khan, 1992, Andreoli \& Khan, 1999; Andreoli \& Khan, 2000). Outro aspecto fisiológico do GA é a indução de aamilase durante o processo de germinação em sementes de cereais, o que implica aumento na germinação da espiga se houver chuvas antes da colheita, o que acarreta a degradação na qualidade da farinha e de cervejas (Lunn et al. 2001).

As vantagens do tratamento biológico em relação ao químico são: a baixa toxicidade, a resistência de alguns patógenos aos fungicidas usados no tratamento de sementes e maior compatibilidade com Rhizobium spp. (Bennett, 1996). Nesse contexto, existem algumas indagações: seria possível a substituição do controle químico pelo biológico? É compensador o tratamento de sementes de alta qualidade? 
Os objetivos deste trabalho foram: avaliar o efeito do condicionamento matricial e da integração deste com o tratamento químico e biológico (Bacillus) das sementes na emergência e na produtividade de milho, bem como o efeito do "priming" na melhoria da qualidade de sementes de baixo vigor.

\section{Material e Métodos}

O trabalho constou de três experimentos descritos, a seguir:

\section{Experimento 1}

Este experimento foi conduzido na Embrapa Milho e Sorgo, em Sete Lagoas, MG, utilizando-se sementes de milho híbrido duplo BRS 201, da Embrapa. Antes da semeadura, realizada em 31 de outubro de 1996, as sementes foram submetidas aos seguintes tratamentos:

1-Testemunha; 2 -Sementes tratadas com $\mathrm{Captan}^{\circledR}(\mathrm{F})$, (cis-N-triclorometiltio-4-ciclohexano1,2-dicarbomixida) a 2,25 g i.a. $\mathrm{kg}^{-1}$ de sementes; 3 - Sementes tratadas com Kodiak ${ }^{\circledast}(\mathrm{K})$, (B. subtilis da Gustafson Inc. Texas, USA) a $600 \mathrm{mg} \mathrm{kg}^{-1} \mathrm{de}$ sementes; 4-Sementes condicionadas com MicroCel E (MC), (silicato de cálcio de rocha diatomáceas da Mainville Inc., Denver, USA), sem secagem; 5 Sementes condicionadas na presença de $\operatorname{Kodiak}^{\circledR} \mathrm{a}$ $600 \mathrm{mg} \mathrm{kg}^{-1}$ de sementes $(\mathrm{K} \rightarrow \mathrm{MC}) ; 6$ - Sementes condicionadas na presença de $\operatorname{Kodiak}^{\circledR}$ a $600 \mathrm{mg}$ $\mathrm{kg}^{-1}$ de sementes e Captan a 2,25 g i.a kg-1 de sementes $(\mathrm{K} \rightarrow \mathrm{MC} \rightarrow \mathrm{F}) ; 7$ - Sementes condicionadas na presença de ácido giberélico $(\mathrm{MC} \rightarrow \mathrm{GA})$, $\left(\mathrm{GA}_{4+7}, 99 \%\right.$ i.a., da Sigma, São Paulo, SP) a 100 $\mu \mathrm{M} ; 8$ - Sementes condicionadas como no tratamento 4 e secadas no laboratório, à temperatura ambiente (MC $\rightarrow$ seca).

Nos tratamentos 4, 5, 6, 7 e 8, as sementes foram condicionadas com Micro-Cel E à razão de 20:5:20 (semente :produto: água), em frasco de vidro de boca larga, por 48 horas, a $22^{\circ}$ C. Após esse período, a mistura foi peneirada e as sementes foram secadas até teor de água de $13 \%$, à sombra, no laboratório de sementes da Embrapa Milho e Sorgo, em Sete Lagoas, MG.

Para a semeadura manual em campo, quatro repetições de 100 sementes de cada tratamento foram distribuídas a uma distância de $20 \mathrm{~cm}$, em quatro linhas de $5 \mathrm{~m}$ de comprimento, espaçadas de 1 $m$ entre si. As parcelas receberam adubação de plantio de $300 \mathrm{~kg} \mathrm{ha}^{-1}$ da fórmula 8: $28: 16$ e $60 \mathrm{~kg} \mathrm{de} \mathrm{N}$ $\mathrm{ha}^{-1}$ em cobertura, aos 35 dias após a semeadura. O delineamento foi o de blocos ao acaso com oito tratamentos e quatro repetições.

Os parâmetros avaliados foram: porcentagem de plântulas emergidas aos 20 dias, índice de velocidade de emergência (IVE), estande final, número de espigas ha ${ }^{-1}$, produção de espigas e de grãos por hectare. As espigas das plantas das quatro linhas foram colhidas, debulhadas e pesadas. O teor de umidade dos grãos foi determinado imediatamente após a colheita e a produção, quantificada em $\mathrm{kg}$ $\mathrm{ha}^{-1}$, foi ajustada para $13 \%$ de umidade.

\section{Experimento 2}

Este trabalho foi desenvolvido na Embrapa Negócios Tecnológicos, em Ponta Grossa, PR. As sementes tratadas e não tratadas dos híbridos BRS 3123, XL 330 e AS 22 foram semeadas em 5 de outubro de 1997.

Os tratamentos para o híbrido BRS 3123 foram: 1 - Testemunha; 2 -Sementes tratadas com Captan ${ }^{\circledR}$ a 2,25 g i.a. kg ${ }^{-1}$ de sementes; 3 - Sementes tratadas com Kodiak ${ }^{\circledR}$ a $600 \mathrm{mg} \mathrm{kg}^{-1}$ de sementes; 4-Sementes condicionadas com Micro-Cel E; 5-Sementes condicionadas na presença de Kodiak $^{\circledR}$ a $600 \mathrm{mg} \mathrm{kg}^{-1}$ de sementes; 6 - Sementes condicionadas na presença de $\operatorname{Kodiak}^{\circledR}$ a 600 mg. $\mathrm{kg}^{-1}$ de sementes e Captan ${ }^{\circledR}$ a 2,25 g i.a. $\mathrm{kg}^{-1}$ de sementes.

Os tratamentos 1, 2 e 3 foram também avaliados nos híbridos XL 330 e AS 22. Nos tratamentos 4,5 , e 6 , as sementes foram condicionadas com 
Micro-Cel E à razão de 20: 5: 20 (semente: produto: água), em frasco de boca larga, por 48 horas, a $22^{\circ} \mathrm{C}$. Após esse período, a mistura foi peneirada e as sementes foram secadas no laboratório. Cinco repetições de 100 sementes foram distribuídas em quatro linhas de $5 \mathrm{~m}$ de comprimento, espaçadas de $1 \mathrm{~m}$. As parcelas receberam adubação de $300 \mathrm{~kg}$ $\mathrm{ha}^{-1}$ da fórmula 8: $28: 16$ e $60 \mathrm{~kg} \mathrm{~N} \mathrm{ha}{ }^{-1}$ em cobertura, aos 35 dias após a semeadura. O delineamento experimental foi o de blocos ao acaso, com seis tratamentos para o híbrido BRS 3123 e três tratamentos para os híbridos XL 330 e AS 22.

Os parâmetros avaliados foram: porcentagem de plântulas emergidas aos 10 e 20 dias, índice de velocidade de emergência (IVE), estande final, número de espigas ha ${ }^{-1}$, a produção de espigas e de grãos por hectare. As espigas das plantas das quatro linhas foram colhidas, debulhadas e pesadas. $\mathrm{O}$ teor de umidade do grão foi determinado imediatamente após a colheita e a produção, quantificada em $\mathrm{kg} \mathrm{ha}^{-1}$,foi ajustada para $13 \%$ de umidade.

\section{Experimento 3}

Dois lotes de sementes do milho híbrido BRS 3123 , com germinação inicial de $95 \%$ e $85 \%$, submetidos a diferentes tratamentos, foram semeados na Embrapa Milho e Sorgo em, Sete Lagoas, MG, em 13 de novembro de 1998.

Os tratamentos foram: 1 - Sementes com germinação de 95\% e sem tratamento; 2 - Sementes com germinação de $85 \%$ e sem tratamento; 3 sementes com germinação de $95 \%$ condicionadas com Micro-Cel E à razão de 20: 5: 20 (semente: produto: água), a $22^{\circ} \mathrm{C}$, por 48 horas; 4 - Sementes com germinação de $85 \%$, condicionadas com Micro-Cel E à razão de 20: 5: 20 (semente: produto: água), a $22^{\circ} \mathrm{C}$, por 48 horas; 5 - sementes com germinação de $95 \%$ condicionadas com Micro-Cel E à razão de 20: 5: 20 (semente: produto: água), a $22^{\circ} \mathrm{C}$, por 48 horas, na presença de $600 \mathrm{mg}$ Bacillus $\mathrm{kg}^{-1}$ de sementes; 6 - sementes com germinação de
$85 \%$ condicionadas com Micro-Cel E à razão de 20: 5: 20 (semente: produto: água), a $22^{\circ} \mathrm{C}$, por 48 horas, na presença de $600 \mathrm{mg}$ Bacillus $\mathrm{kg}^{-1}$ de sementes.

Logo após o tratamento das sementes, quatro repetições de 100 sementes foram distribuídas em quatro linhas de $5 \mathrm{~m}$ de comprimento e espaçadas de $0,9 \mathrm{~m}$. As parcelas receberam adubação de $300 \mathrm{~kg} \mathrm{ha}^{-1}$ da fórmula 8: 28: 16 e $60 \mathrm{~kg} \mathrm{~N} \mathrm{ha}^{-1} \mathrm{em}$ cobertura, aos 35 dias após a semeadura. $\mathrm{O}$ delineamento experimental foi o de blocos ao acaso, com quatro repetições.

Os parâmetros avaliados foram: porcentagem de plântulas emergidas aos 20 dias, índice de velocidade de emergência (IVE), $\mathrm{T}_{50}$ (o tempo quando a porcentagem de germinação atingiu 50\%), estande final, produção de espigas e produção de grãos por hectare. As espigas das plantas das quatro linhas foram colhidas, debulhadas e pesadas. $\mathrm{O}$ teor de umidade do grão foi determinado imediatamente após a colheita e a produção, quantificada em $\mathrm{kg} \mathrm{ha}^{-1}$, foi ajustada para $13 \%$ de umidade.

As análises estatísticas dos experimentos foram realizadas com o programa MSTAT (Michigan State University, East Leasing, MI) e as comparações entre as médias foram efetuadas pelo teste de Duncan, a 5\% de probabilidade.

\section{Resultados e Discussão}

No experimento 1 , os tratamentos químico e biológico das sementes afetaram $(\mathrm{P}<0,05)$ o número de plantas ha- ${ }^{-1}$ o número de espigas ha ${ }^{-1}, \mathrm{o}$ peso de espigas e a produção de grãos de milho (Tabela 1). As sementes tratadas com Captan ${ }^{\circledR}$, com Kodiak $^{\circledR}$ (Bacillus), condicionadas com Micro Cel (MC) e as condicionadas na presença de GA (MC $\rightarrow$ GA) proporcionaram maiores populações de plantas em relação à testemunha, mas não diferiram das sementes condicionadas com Kodiak ${ }^{\circledR}(\mathrm{K} \rightarrow \mathrm{MC}) \mathrm{e}$ $\operatorname{Kodiak}^{\circledast}$ mais $\operatorname{Captan}^{\circledast}(\mathrm{K} \rightarrow \mathrm{MC} \rightarrow \mathrm{F})$. O número 
TABELA 1. Efeito do matricondicionamento integrado com Bacillus, GA e fungicida na emergência, número de espiga ha ${ }^{-1}$, e na produção de milho BRS 201, em Sete Lagoas, MG. Semeadura em 31 de outubro de 1996.

\begin{tabular}{|c|c|c|c|c|}
\hline \multirow{2}{*}{ Tratamento } & N. ${ }^{0}$ de plantas ha ${ }^{-1}$ & $N^{.0}$ de espiga ha ${ }^{-1}$ & Peso de espigas & Peso de grãos \\
\hline & $(1000)$ & $(1000)$ & $\left(\mathrm{kg} \mathrm{ha}^{-1}\right)$ & $\left(\mathrm{kg} \mathrm{ha}^{-1}\right)$ \\
\hline Testemunha & $55,6 b^{*}$ & $47,6 d^{*}$ & $6.266 \mathrm{c}^{*}$ & $5.140 \mathrm{c}^{*}$ \\
\hline Captan ${ }^{\circledR}(\mathrm{F})$ & 57,4 a & $55,0 \mathrm{ab}$ & $7.696 \mathrm{a}$ & $6.290 \mathrm{a}$ \\
\hline Bacillus (K) & $58,4 \mathrm{a}$ & $50,4 \mathrm{c}$ & $6.614 \mathrm{bc}$ & $5.340 \mathrm{bc}$ \\
\hline $\mathrm{MC}$ & $58,2 \mathrm{a}$ & $57,0 \mathrm{a}$ & $7.190 \mathrm{ab}$ & $5.984 \mathrm{ab}$ \\
\hline $\mathrm{K} \rightarrow \mathrm{MC}$ & $57,2 \mathrm{ab}$ & $54,4 \mathrm{ab}$ & $7.254 \mathrm{ab}$ & $5.956 \mathrm{ab}$ \\
\hline $\mathrm{K} \rightarrow \mathrm{MC} \rightarrow \mathrm{F}$ & $57,2 \mathrm{ab}$ & $52,4 \mathrm{bc}$ & $6.556 \mathrm{bc}$ & $5.384 \mathrm{bc}$ \\
\hline $\mathrm{MC} \rightarrow \mathrm{GA}$ & $58,8 \mathrm{a}$ & $50,4 \mathrm{c}$ & $6.670 \mathrm{bc}$ & $5.408 \mathrm{bc}$ \\
\hline $\mathrm{MC} \rightarrow \mathrm{Seca}$ & $56,6 \mathrm{~b}$ & $55,2 \mathrm{ab}$ & $7.176 \mathrm{ab}$ & $5.900 \mathrm{ab}$ \\
\hline Média & 57,4 & 52,8 & 6.927 & 5.675 \\
\hline
\end{tabular}

F - sementes tratadas com Captan ${ }^{\circledR}$ a 2,25 g i.a. kg-1 de sementes; $\mathrm{K}$ - sementes tratadas com Kodiak (Bacillus subtilis) a $600 \mathrm{mg} \mathrm{kg}^{-1}$ de sementes; $\mathrm{MC}$ - sementes condicionadas com Micro Cel; $\mathrm{K} \rightarrow \mathrm{MC}$ - sementes tratadas com Bacillus, depois condicionadas como descrito anteriormente; $\mathrm{K} \rightarrow \mathrm{MC} \rightarrow \mathrm{F}$ - sementes tratadas com Bacillus, depois condicionadas na presença de $\operatorname{Captan}^{\circledR} ; \mathrm{MC} \rightarrow \mathrm{GA}$ - sementes condicionadas na presença de ácido giberélico a $100 \mu \mathrm{M}$; $\mathrm{MC} \rightarrow \mathrm{seca}$ - sementes condicionadas com Micro-Cel E e secadas no laboratório, à umidade original.

* As médias dentro de cada coluna, seguidas da mesma letra, não diferem entre si pelo teste de Duncan, a 5\% de probabilidade.

de espigas aumentou $(\mathrm{P}<0,05)$ com todos os tratamentos. O condicionamento matricial (MC) produziu o maior número de espigas, mas não diferiu dos tratamentos com Captan ${ }^{\circledast}$, Captan $^{\circledast}$ condicionada com $\mathrm{MC}(\mathrm{K} \rightarrow \mathrm{MC})$ e sementes secadas após o condicionamento ( $\mathrm{MC} \rightarrow \mathrm{seca})$. As sementes tratadas com Bacillus e $\mathrm{MC} \rightarrow \mathrm{GA}$ proporcionaram o menor número de espigas $(\mathrm{P}<0,05)$, embora diferindo da testemunha. $\mathrm{O}$ tratamento de sementes com Captan (F), o (MC), a interação de $\mathrm{MC}$ com Bacillus $(\mathrm{K} \rightarrow \mathrm{MC})$ e sementes secadas após condicionamento ( $\mathrm{MC} \rightarrow$ seca) aumentaram o peso de espigas e de grãos $(\mathrm{P}<0,05)$. Esses tratamentos proporcionaram um aumento na produção de grãos de milho BRS 201 da ordem de $22,8 \%, 14,7 \%, 15,8 \%$ e $14,5 \%$, respectivamente, em relação às sementes não tratadas.

No experimento 2, o tratamento químico (F) e o "biopriming" ( $\mathrm{K} \rightarrow \mathrm{MC}$ e $\mathrm{K} \rightarrow \mathrm{MC} \rightarrow \mathrm{F})$ foram efetivos em aumentar no milho híbrido BRS 201 $(\mathrm{P}<0,05)$ o índice de velocidade de emergência (IVE) e a porcentagem de emergência de plântulas aos dez dias (Tabela 2). Entretanto, não houve diferenças entre os tratamentos $(\mathrm{P}<0,05)$, para o estande final.

As plantas do híbrido XL 330 apresentaram maior desenvolvimento inicial $(\mathrm{P}<0,05)$ e o híbrido AS 22 teve o pior desempenho em relação ao 
TABELA 2. Efeitos do matricondicionamento integrado com tratamento químico e biológico na emergência de plântulas e no índice de velocidade de emergência (IVE) em sementes de milho híbrido BRS 3123, em Ponta Grossa, PR. Semeadura em 5 de outubro de 1997.

\begin{tabular}{lccc}
\hline Tratamento & Emergência 10 dias (\%) & Estande final (\%) & IVE \\
\hline Testemunha & $60,0 \mathrm{~b}^{*}$ & $86,9 \mathrm{a}^{*}$ & $8,25 \mathrm{~b}^{*}$ \\
Captan${ }^{\circledR}(\mathrm{F})$ & $68,4 \mathrm{a}$ & $90,6 \mathrm{a}$ & $8,76 \mathrm{a}$ \\
Bacillus (K) & $66,3 \mathrm{ab}$ & $89,3 \mathrm{a}$ & $8,55 \mathrm{ab}$ \\
$\mathrm{MC}$ & $69,4 \mathrm{a}$ & $86,7 \mathrm{a}$ & $8,50 \mathrm{ab}$ \\
$\mathrm{K} \rightarrow \mathrm{MC}$ & $75,0 \mathrm{a}$ & $89,5 \mathrm{a}$ & $8,81 \mathrm{a}$ \\
$\mathrm{K} \rightarrow \mathrm{MC} \rightarrow \mathrm{F}$ & $75,0 \mathrm{a}$ & $89,4 \mathrm{a}$ & $8,80 \mathrm{a}$ \\
\hline Média & 69,0 & 88,7 & 8,61 \\
\hline
\end{tabular}

$\mathrm{F}$ - sementes foram tratadas com Captan ${ }^{\circledR}$ a 2,25 g i.a. $\mathrm{kg}^{-1}$ de sementes; K sementes tratadas com Kodiak (Bacillus subtilis) a $600 \mathrm{mg} \mathrm{kg}^{-1}$ de sementes; $\mathrm{MC}$ - sementes condicionadas com Micro Cel; $\mathrm{K} \rightarrow \mathrm{MC}$ - sementes tratadas com Bacillus, depois condicionadas como anteriormente; $\mathrm{K} \rightarrow \mathrm{MC} \rightarrow \mathrm{F}$ - sementes tratadas com Bacillus, depois condicionadas na presença de Captan ${ }^{\circledR}$.

*As médias dentro das colunas, seguidas da mesma letra, não diferem entre si pelo teste de Duncan, a 5\% de probabilidade.

arranque inicial $(\mathrm{P}<0,05)$, nas condições de solo frio e úmido de Ponta Grossa, conforme mostra a porcentagem de emergência de plântulas aos dez dias após a semeadura e o índice de velocidade de emergência (Tabela 3 ). Nesse período, a temperatura mínima variou de 5 a $8^{\circ} \mathrm{C}$. Para o híbrido XL330, os tratamentos químico e biológico não favoreceram a emergência ou o IVE aos dez dias $(\mathrm{P}<0,05)$. Entretanto, o tratamento químico foi efetivo em aumentar a emergência e o IVE para o híbrido AS 22 $(\mathrm{P}<0,05)$. Para os dois híbridos, a emergência aos vinte dias e o estande final apresentaram o mesmo comportamento (Tabela 3). Para o híbrido AS 22 tratado com Captan ${ }^{\circledR}$ ocorreram diferenças entre a emergência aos vinte dias, estande final e o IVE, o que pode ser atribuído à menor lixiviação de exsudatos (Khan et al.,1995; Andreoli \& Khan, 2000).

Não houve diferença $(\mathrm{P}<0,05)$ entre os híbridos BRS 3123, XL 330 e AS 22 no peso de espigas, no peso de grãos e na população de plantas na colheita, exceto para o número de espigas (Tabelas 4 e 5). A semente do híbrido BRS 3123 (Tabela 4) tratada com Bacillus (K), condicionada com Micro-Cel E (MC) e tratada com Bacillus (K), depois condicionada na presença de $\operatorname{Captan}^{\circledast}(\mathrm{K} \rightarrow$ $\mathrm{MC} \rightarrow \mathrm{F}$ ), propiciou o aumento do número de espigas, mas não a produtividade $(\mathrm{P}<0,05)$. Fato semelhante ocorreu com o AS22 (Tabela 5), quando as sementes foram tratadas com Captan ${ }^{\circledR}(\mathrm{F})$. Isso demonstra que o efeito dos tratamentos químicos e biológicos no início do estabelecimento da cultura não refletiu em ganhos proporcionais aos rendimentos. Esses dados concordam com os de Andreoli \& Khan (1999; 2000); Callan et al.(1990); Parera \& Cantliffe (1992). Isso significa que os tratamentos de sementes tiveram efeitos temporais na emergência e no estabelecimento da cultura, mas não proporcionaram mudanças fisiológicas que tenham refletido em ganhos de produtividade. 
TABELA 3. Efeitos dos tratamentos químico e biológico na emergência de plântulas aos dez dias e vinte dias, estande final e no índice de velocidade de emergência (IVE), em dois genótipos de milho híbrido, em Ponta Grossa, PR. Semeadura em 5 de outubro de 1997.

\begin{tabular}{lccccc}
\hline Genótipo & Tratamento & $\begin{array}{c}\text { Emergência 10 } \\
\text { dias (\%) }\end{array}$ & $\begin{array}{c}\text { Emergência 20 } \\
\text { dias (\%) }\end{array}$ & Estande Final (\%) & IVE \\
\hline \multirow{2}{*}{ XL 330 } & Testemunha & $74,0 \mathrm{ab}^{*}$ & $87,1 \mathrm{ab}^{*}$ & $86,2 \mathrm{ab}^{*}$ & $8,52 \mathrm{ab}^{*}$ \\
& Captan® (F) & $79,8 \mathrm{a}$ & $90,5 \mathrm{a}$ & $89,9 \mathrm{a}$ & $8,86 \mathrm{a}$ \\
& Bacillus (K) & $70,7 \mathrm{ab}$ & $84,3 \mathrm{~b}$ & $83,9 \mathrm{~b}$ & $8,21 \mathrm{~b}$ \\
& Média & 74,8 & 87,3 & 86,7 & 8,53 \\
\hline \multirow{2}{*}{ AS 22 } & Testemunha & $48,0 \mathrm{~b}$ & $80,9 \mathrm{~b}$ & $80,2 \mathrm{~b}$ & $7,55 \mathrm{~b}$ \\
& Captan® (F) & $65,3 \mathrm{ab}$ & $88,2 \mathrm{a}$ & $87,8 \mathrm{a}$ & $8,50 \mathrm{a}$ \\
& Bacillus (K) & $46,3 \mathrm{~b}$ & $80,4 \mathrm{~b}$ & $79,9 \mathrm{~b}$ & $7,48 \mathrm{~b}$ \\
& Média & 53,2 & 83,2 & 82,6 & 7,84 \\
\hline
\end{tabular}

F - sementes tratadas com Captan $^{\circledR}$ a 2,25 g i.a. kg ${ }^{-1}$ de sementes; $\mathrm{K}$ - sementes tratadas com Kodiak (Bacillus subtilis) a $600 \mathrm{mg} \mathrm{kg}^{-1}$ de sementes;

*As médias dentro das colunas, seguidas da mesma letra, não diferem entre si pelo teste de Duncan, a $5 \%$ de probabilidade

TABELA 4. Efeitos do matricondicionamento integrado com tratamento químico e biológico no número de espigas ha ${ }^{-1}$, na produção de espigas e na produção de grãos, em milho híbrido BRS3123, em Ponta Grossa, PR. Semeadura em 5 outubro de 1997.

\begin{tabular}{|c|c|c|c|c|c|}
\hline Genótipo & Tratamento & $\begin{array}{l}N^{\circ} \text { de espiga } \\
\text { ha }^{-1}(1000)\end{array}$ & $\begin{array}{l}\text { Peso de espigas } \\
\left(\mathrm{Kg} \mathrm{ha}^{-1}\right)\end{array}$ & $\begin{array}{l}\text { Peso de grãos } \\
\left(\mathrm{Kg} \mathrm{ha}^{-1}\right)\end{array}$ & $\begin{array}{c}\text { Plantas na } \\
\text { colheita (\%) }\end{array}$ \\
\hline \multirow{6}{*}{ BRS 3123} & Testemunha & $80,8 b^{*}$ & $10.491,3 \mathrm{a}^{*}$ & $8.803,6 \mathrm{a}^{*}$ & $79,8 a^{*}$ \\
\hline & Captan ${ }^{\circledR}(\mathrm{F})$ & $84,6 \mathrm{ab}$ & $10.217,2 \mathrm{a}$ & $8.535,1 \mathrm{a}$ & $80,8 \mathrm{a}$ \\
\hline & Bacillus (K) & $85,8 \mathrm{a}$ & $9.880,8 \mathrm{a}$ & $8.342,0 \mathrm{a}$ & $75,2 \mathrm{a}$ \\
\hline & $\mathrm{MC}$ & $90,6 \mathrm{a}$ & $10.640,8 \mathrm{a}$ & $8.884,0 \mathrm{a}$ & $78,4 \mathrm{a}$ \\
\hline & $\mathrm{K} \rightarrow \mathrm{MC}$ & $84,6 \mathrm{ab}$ & $10.547,4 \mathrm{a}$ & $8.672,2 \mathrm{a}$ & $74,0 \mathrm{a}$ \\
\hline & $\mathrm{K} \rightarrow \mathrm{MC} \rightarrow \mathrm{F}$ & $86,4 \mathrm{a}$ & $10.397,8 \mathrm{a}$ & $8.709,5 \mathrm{a}$ & $81,4 \mathrm{a}$ \\
\hline & Média & 85,5 & $10.362,5$ & $8.657,7$ & 78,3 \\
\hline
\end{tabular}

$\mathrm{F}$ - sementes tratadas com Captan $^{\circledR}$ a 2,25 g i.a kg-1 de sementes; $\mathrm{K}$ - sementes tratadas com Kodiak (Bacillus subtilus) a $600 \mathrm{mg} \mathrm{kg}^{-1}$ de sementes; MC- sementes condicionadas com Micro Cel; $\mathrm{K} \rightarrow \mathrm{MC}$ - sementes tratadas com Bacillus, depois condicionadas como anteriormente; $\mathrm{K} \rightarrow \mathrm{MC} \rightarrow \mathrm{F}$ - sementes tratadas com Bacillus, depois condicionadas na presença de Captan ${ }^{\circledR}$.

*As médias dentro das colunas, seguidas da mesma letra, não diferem entre si pelo teste de Duncan, a 5\% de probabilidade 
TABELA 5. Efeitos do tratamento químico e biológico no número de espigas ha ${ }^{-1}$, na produção de espigas e na produção de grãos ha ${ }^{-1}$,em dois milho híbridos, em Ponta Grossa, PR. Semeadura em 5 de outubro de 1997.

\begin{tabular}{|c|c|c|c|c|c|}
\hline Genótipo & Tratamento & $\begin{array}{c}N^{\circ} \text { de espiga } \\
\text { ha }^{-1}(1000)\end{array}$ & $\begin{array}{c}\text { Peso de espigas } \\
\left(\mathrm{Kg} \mathrm{ha}^{-1}\right)\end{array}$ & $\begin{array}{c}\text { Peso de grãos } \\
\left(\mathrm{Kg} \mathrm{ha}^{-1}\right)\end{array}$ & $\begin{array}{l}\text { Plantas na } \\
\text { colheita (\%) }\end{array}$ \\
\hline \multirow{3}{*}{ XL 330} & Testemunha & $93,2 \mathrm{ab}^{*}$ & $10.279,5 \mathrm{a}^{*}$ & $8.628,5 \mathrm{a}^{*}$ & $78,0 a^{*}$ \\
\hline & Captan $®(F)$ & $97,8 \mathrm{a}$ & $10.416,6 \mathrm{a}$ & $8.759,4 \mathrm{a}$ & $80,4 \mathrm{a}$ \\
\hline & Bacillus (K) & $89,0 \mathrm{~b}$ & $10.217,2 \mathrm{a}$ & $8.535,1 \mathrm{a}$ & $82,4 \mathrm{a}$ \\
\hline \multirow{6}{*}{ AS 22} & Média & 93,3 & $10.304,4$ & $8.641,0$ & 80,3 \\
\hline & Testemunha & $76,2 \mathrm{~b}$ & $9.569,3 \mathrm{a}$ & $7.706,5 \mathrm{~b}$ & $73,4 \mathrm{a}$ \\
\hline & Captan $®(F)$ & $86,0 \mathrm{a}$ & $10.067,7 \mathrm{a}$ & $8.111,5 \mathrm{ab}$ & $79,4 \mathrm{a}$ \\
\hline & Bacillus (K) & $76,8 \mathrm{~b}$ & $9.862,1 \mathrm{a}$ & $7.912,1 \mathrm{~b}$ & $71,6 \mathrm{a}$ \\
\hline & Média & 79,7 & $9.833,0$ & $7.910,0$ & 74,8 \\
\hline & $\mathrm{CV}(\%)$ & 7,80 & 7,05 & 7,70 & 6,76 \\
\hline
\end{tabular}

F - sementes tratadas com Captan ${ }^{\circledR}$ a 2,25 g i.a. kg $^{-1}$ de sementes; $\mathrm{K}$ - sementes tratadas com Kodiak ${ }^{\circledR}$ (Bacillus subtilus) a $600 \mathrm{mg} \mathrm{kg}^{-1}$ de sementes.

*As médias dentro das colunas seguidas da mesma letra não diferem entre si pelo teste de Duncan a $5 \%$ de probabilidade.

No experimento 3 , houve diferença significativa $(\mathrm{P}<0,05)$ na qualidade inicial da semente $(\mathrm{Ta}-$ bela 6 e Figura 1). A porcentagem média do estande final para as sementes Q1 (85\% de germinação) e Q2 (95\% de germinação) foi 69,9 e 85,5\%, respectivamente. Os tratamentos fisiológico e biológico não aumentaram $(\mathrm{P}>0,05)$ o estande final para as sementes de alta qualidade $(95 \%)$, mas houve efeito $(\mathrm{P}<0,05)$ dos tratamentos para as sementes de menor qualidade (85\%), quando comparado com a semente não tratada. $\mathrm{O}$ estande estabelecido com sementes de menor qualidade aumentou de $60,8 \%$ (sementes não tratadas) para 74,4\% com $\mathrm{MC}, \mathrm{o}$ que representa um aumento de $22,4 \%(\mathrm{P}<0,05)$. Entretanto, não houve incremento em relação ao $\mathrm{MC}$ com a adição de Bacillus. Houve vantagem de 1,8 dia na velocidade de emergência $(\mathrm{P}<0,05)$, avaliada pelo $\mathrm{T}_{50}$, para as sementes de menor qualidade
(85\%) condicionadas com MC, quando comparou com o controle (Tabela 6 e Figura 1).

Houve aumento no número de espigas ha ${ }^{-1}$ $(\mathrm{P}<0,05)$ quando sementes de menor qualidade (85\%) foram condicionadas com MC e MC na presença de Bacillus, mas não houve resposta $(\mathrm{P}<0,05)$ para as sementes de alta qualidade (Tabela 6). Isso sugere que durante os tratamentos fisiológicos (MC) e "biocondicionamento" $(\mathrm{MC} \rightarrow \mathrm{K})$, as organelas e as membranas das sementes deterioradas devido ao envelhecimento foram reparadas e reorganizadas, bem como os eventos bioquímicos e fisiológicos associados com a rapidez e a sincronia da germinação retomados o que, restabeleceu o vigor das sementes (Khan, 1992; Khan \& Andreoli, 1993; Andreoli \& Khan, 2000).

$\mathrm{O}$ condicionamento matricial e sua integração com o tratamento biológico $(\mathrm{MC} \rightarrow \mathrm{K})$ 
TABELA 6. Efeito da qualidade de semente do híbrido BRS3123, do matricondicionamento e MC+Bacillus no estande final, $\mathrm{T}_{50}$, número de espiga ha ${ }^{-1}$, produtividade e peso de 1000 grãos de milho, em Sete Lagoas, MG. Semeadura em 13 de novembro de 1998.

\begin{tabular}{|c|c|c|c|c|c|c|c|}
\hline $\begin{array}{l}\text { Qualidade da } \\
\text { semente (\%) }\end{array}$ & Tratamento & $\begin{array}{c}\text { Estande final } \\
(\%)\end{array}$ & $\mathbf{T}_{50}$ & $\begin{array}{c}\mathrm{N}^{\circ} \text { de espigas } \\
\mathrm{ha}^{-1}(\mathbf{1 0 0 0 )}\end{array}$ & $\begin{array}{c}\text { Peso de espigas } \\
\left(\mathrm{t} \mathrm{ha}^{-1}\right)\end{array}$ & $\begin{array}{l}\text { Peso de grãos } \\
\left(\mathrm{t} \mathrm{ha}^{-1}\right)\end{array}$ & $\begin{array}{l}\text { Peso } 1000 \\
\text { grãos (g) }\end{array}$ \\
\hline \multirow{3}{*}{ Q1 (85) } & Controle & $60,8 b^{*}$ & $5,62 \mathrm{~d}$ & $67,2 c^{*}$ & $8,2 \mathrm{~d}^{*}$ & $6,9 \mathrm{~d}^{*}$ & $250,9 a^{*}$ \\
\hline & $\mathrm{MC}$ & $74,4 \mathrm{a}$ & $3,80 \mathrm{a}$ & $82,0 \mathrm{~b}$ & $9,2 \mathrm{~b}$ & $7,9 \mathrm{c}$ & $234,9 \mathrm{a}$ \\
\hline & $\mathrm{MC}+\mathrm{K}$ & $74,6 \mathrm{a}$ & $3,82 \mathrm{~b}$ & $80,4 \mathrm{~b}$ & $9,0 \mathrm{~b}$ & $7,6 \mathrm{c}$ & $23,9 \mathrm{a}$ \\
\hline \multirow{6}{*}{ Q2 (95) } & Média & 69,9 & 4,41 & 76,5 & 8,8 & 7,5 & 238,9 \\
\hline & Controle & $84,6 \mathrm{ab}^{*}$ & $4,60 \mathrm{c}$ & $92,8 \mathrm{a}$ & $10,6 \mathrm{a}$ & $8,9 \mathrm{ab}$ & $240,5 \mathrm{a}$ \\
\hline & $\mathrm{MC}$ & $87,4 \mathrm{a}$ & $3,40 \mathrm{a}$ & $92,0 \mathrm{a}$ & $10,0 \mathrm{a}$ & $8,3 \mathrm{~b}$ & $235,2 \mathrm{a}$ \\
\hline & $\mathrm{MC}+\mathrm{K}$ & $84,6 \mathrm{ab}$ & $3,66 \mathrm{ab}$ & $91,8 \mathrm{a}$ & $11,3 \mathrm{a}$ & $9,5 \mathrm{a}$ & $248,2 \mathrm{a}$ \\
\hline & Média & 85,5 & 3,88 & 92,2 & 10,6 & 8,9 & 241,3 \\
\hline & $\mathrm{CV}(\%)$ & 9,65 & 4,56 & 7,93 & 10,66 & 9,8 & 6,03 \\
\hline
\end{tabular}

Q1 - lote de semente com $85 \%$ de germinação; Q2 - lote de semente com $95 \%$ de germinação; $\mathrm{MC}$ - sementes condicionadas com Micro-Cel E; MC+K - sementes condicionadas na presença de $600 \mathrm{mg}$ de Bacillus $\mathrm{kg}^{-1}$ de sementes.

*As médias dentro das colunas, seguidas da mesma letra, não diferem significativamente pelo teste de Duncan, a 5\% de probabilidade.

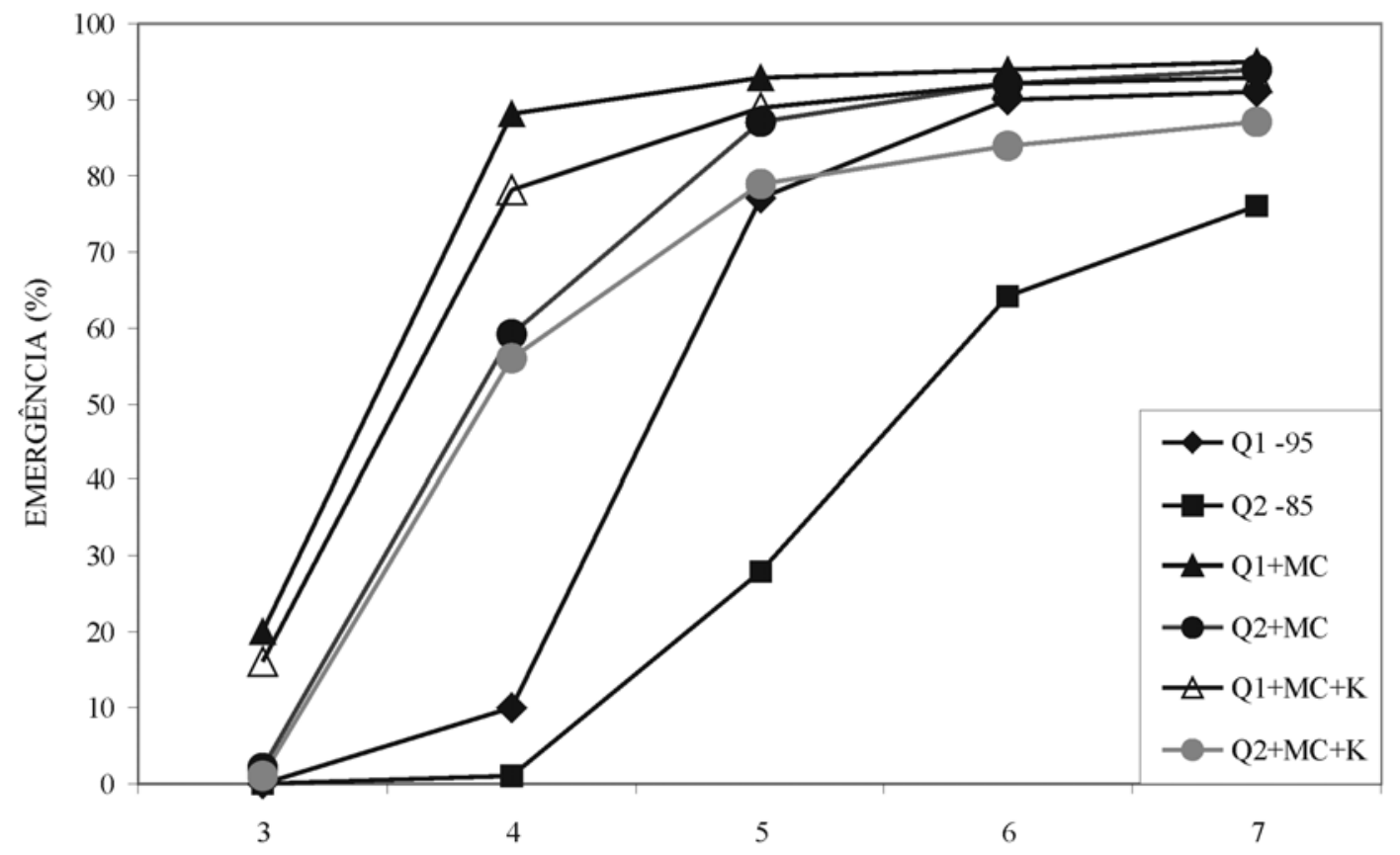

DIAS APÓS O PLANTIO

FIGURA 1. Efeito da qualidade das sementes (Q1) e (Q2) condicionadas com Micro Cel E (MC) e MC na presença de Bacillus $(\mathrm{MC}+\mathrm{K})$ na emergência final e no índice de velocidade de emergência $\left(\mathrm{T}_{50}\right)$, em milho BRS 3123, em Sete Lagoas, MG, em 1998/1999. As barras indicam diferenças significativas entre as médias, pelo teste de Duncan, a 5\% de probabilidade. 
foram efetivos $(\mathrm{P}<0,05)$ em aumentar o peso de espigas e de grãos em relação às sementes não tratadas e de menor vigor (85\%) (Tabela 6). O aumento da produtividade de grãos foi da ordem de $14,5 \%$ no tratamento MC. Os tratamentos não afetaram $(\mathrm{P}<0,05)$ o peso de 1.000 sementes.

Os tratamentos fisiológicos e biológicos foram efetivos $(\mathrm{P}<0,05)$ na melhoria da qualidade de semente, especialmente nas condições de estresses de Ponta Grossa e, conseqüentemente, no aumento da população de plantas e da produtividade de milho. Esses resultados corroboram os de Andreoli et al. (2002). O "biopriming” foi tão eficaz quanto o tratamento químico (F) no estabelecimento da cultura e na produtividade de milho, o que indica que o "biopriming" poderia substituir o tratamento das sementes de milho com o fungicida Captan ${ }^{\circledR}$.

\section{Conclusões}

A qualidade da semente é fundamental no estabelecimento da cultura e na produtividade de milho.

Semente de alta qualidade não responde aos tratamentos de condicionamento.

Nas condições de estresse, os tratamentos químico e "biopriming" aumentam a qualidade da semente e a produtividade de milho.

A integração do condicionamento matricial (MC) com o tratamento biológico (Bacillus) é efetiva em aumentar o vigor das sementes de milho de menor qualidade.

\section{Agradecimentos}

ADr. Pedro Abel Vieira, da Embrapa/SNT, Sete Lagoas, MG, e a Antônio Lúcio França Perez, do Laboratório de Sementes da Embrapa Milho e Sorgo, Sete Lagoas, MG o apoio na execução deste trabalho.

\section{Literatura Citada}

ANDREOLI, C. Matriconditioning integrated with chemical and biological treatments to improve germination and stand establishment of tomato, eggplant and sweet corn. In: INTERNATIONAL SYMPOSIUM OF THE ISHS, 9, Piracicaba, 2001. On timing of field production in vegetable crops. Piracicaba: ESALQ/USP, 2001. v.1, p.18.

ANDREOLI, C.; KAHN, A.A. Improving seedling emergence of papaya, Carica papaya L., by combining matriconditioning and gibberellin treatment. HortScience, Alexandria, v. 28, p. 708 - 709, 1993.

ANDREOLI, C.; KHAN, A.A. Matriconditioning integrated with gibberellic acid to hasten seed germination and improve stand establishment of pepper and tomato. Pesquisa Agropecuária Brasileira, Brasília, v .34, p. 1953 - 1958, 1999.

ANDREOLI, C.; KHAN, A.A. Integration of physiological, chemical and biological seed treatments to improve stand establishment and yield of vegetables. Acta Horticulturae, The Hague, n. 533, p. $31-38,2000$.

ANDREOLI, C.; ANDRADE, R.V.; ZAMORA, D.; GORDON, M. Influência da qualidade da semente e da densidade de semeadura no estabelecimento e na produtividade de milho. Revista Brasileira de Sementes, Brasília, v. 24, n. 2, p. 1 - 5. 2002.

BENNETT, M.A. Biological seed treatments for improved corn germination and emergence. In: ANNUAL CORN AND SORGHUM RESEARCH CONFERENCE, 51., 1996, Washington. Proceedings. Washington: American Seed Trade Association, 1996. p. 200 - 211.

CALLAN, N.W.; MATHRE, D.E.; MILLER, J.B. Biopriming seed treatment for biological control of Phytium ultimum preemergence damping-off in sh2 sweet corn. Plant Disease, St. Paul, v. 74, p. 368 - 372, 1990. 
CALLAN, N.W.; MATHRE, D.E.; MILLER, J.B. Field performance of sweet corn seed bio-primed and coated with Pseudomonas fluorescens AB254. HortScience,Alexandria, v. 26, p. 1163 - 1165, 1991.

KHAN, A.A. Preplant physiological seed conditioning. Horticultural Reviews, West Post, v. 13, p. 131 - 181, 1992.

KHAN, A.A.; ANDREOLI, C. Hormonal control of seed dormancy and germination under stressful and non-stresful conditions. In: INTERNATIONAL WORKSHOP ON SEEDS, 4., 1992, Angers. Basic and applied aspects of seed biology. $\mathrm{Pa}$ ris: Université Pierre et Marie Curie, 1993. v. 2, p. $625-632$.

KHAN, A.A.; ILYAS, S.; PTASZNIK, W. Integrating low water potential seed hydration with others treatments to improve cold tolerance. Annals of Botany, London, v.75, p. 13 -19, 1995.

KUBIK, K.K.; EASTIN, J.A.; EASTIN, J.D.; ESKRIDGE, K.M. Solid matrix priming of tomato and pepper. In: NATIONAL SYMPOSIUM STAND ESTABLISHMENT HORTICULTURAL CROPS, 6., 1988, Lancaster. Proceedings. Lancaster, PA.1988 p.86-96.
LUNN, G. D.; MAJOR, B. J.; KETTLEWELL, P.S.; SCOTT, R. K. Mechanisms leading to excess alpha-amylase activity in wheat (Triticum aestivum, L) grain in the U.K. Journal of Cereal Science, London, v. 33, p. 313 - 329, 2001.

PARERA, C.A.; CANTLIFFE, D.J. Enhanced emergence and seedling vigor in shrunken-2 sweet corn via seed desinfection and solid matrix priming. Journal of the American Society for Horticultural Science, Mount Vernon, v. 117, p. 400 - 403, 1992.

ROWSE, H.R. Drum priming - a non-osmotic method of priming seeds. Seed Science \& Technology, Zurick, v. 24, p. 281 - 294, 1996.

TAYLOR, A.G.; KLEIN, D.E.; WHITLOW, T.H. Solid matrix priming of seed. Scientia Horticulturae, Amrterdam, v. 37, p. 1 - 11, 1988.

TAYLOR, A.G.; ALLEN, P.S.; BENNETT, M.A.; BRADFORD, K.J.; BURRIS, J.S.; MISRA, M.K. Seed enhancements. Seed Science Research, Oxon, v. 8, p. 245 - 256, 1998.

WARREN, J.E.; BENNETT, M.A. Seed hydration using the drum priming system. HortScience, Alexandria, v. 32,p. 1220 - 1221, 1997. 